\title{
Editorial
}

\section{"Crisis energética": La energía nuclear, ¿una alternativa complementaria?}

Utilizamos la expresión "crisis" en su doble sentido etimológico; reconocemos que la situación es grave y buscamos quién nos dé una solución. Cuando el editorial del semanario Proceso, $N^{\circ} 1142$, 26 abril 2005, habla de "crisis energética", está haciendo referencia al largo proceso de crecimiento frenado con alza de precios, derivado fundamentalmente de los elevados precios del petróleo, que afecta tanto a las economías centroamericanas como a la economía de Estados Unidos, de acuerdo a su propio presidente. La palabra crisis y "critica" significan también el acto de juzgar, evaluar, ponderar, es decir, buscar las razones y proponer las soluciones a determinada situación caótica. El resultado bistórico es una prolongada crisis energética mundial desde los años 70, acompañada de una imperfecta crítica que analiza y conoce las razones pero que se resiste a poner o imponer las soluciones. Esta parece ser la contradicción interna de la crisis energética: la resistencia a aplicar soluciones de largo plazo en razón de intereses económicos de más corto plazo. La propuesta de la energía nuclear, como una 
alternativa complementaria al petróleo, no proviene de un astrofísico sino del presidente George W. Bush en su discurso a la nación del 28 de abril 2005.

\section{Breve bistoria de la crisis energética}

Haciendo una breve memoria, en 1973 los gobiernos de la OPEP, paises productores y exportadores de petróleo, cuadruplican el precio del 'oro negro' porque las naciones industrializadas lo babian adquirido $y$ derrochado en la onda larga creciente del Kondratief 1950-1973. Los precios bajos invitaban al derroche del petróleo cuando en 1972 se publica el informe de Club de Roma: "Los límites del crecimiento económico". "Si se mantenían los niveles de crecimiento poblacional, industrialización, contaminación, producción de alimentos y agotamiento de recursos, los limites del crecimiento se darian en los cien próximos años". Después de la publicación del informe del Club de Roma se disparan en los países occidentales los precios del petróleo y de las materias primas.

Todos los países no productores de petróleo van a enfrentar una grave crisis. Aqui en El Salvador el impacto fue muy fuerte porque se habia iniciado poco antes el Mercado Común, entrecortado durante diez años con la Guerra de 100 boras, de manera que la brusca alza del petróleo bizo que el nivel de precios 1972=100 se duplicara para 1978; bubo que buscar una solución parcial en la represa del Cerrón Grande. Nuestro gobierno optó por la alternativa bidroeléctrica; las grandes compañias de automóviles se especializaron en carros compactos, menos devoradores del crudo; los países del Norte buscaron otros combustibles para la calefacción doméstica y algunos sustituyeron el petróleo por el carbón de Polonia.

Sin embargo, el susto fue relativamente de corto plazo "porque se estimuló la explotación de nuevos yacimientos y la investigación de técnicas aborradoras de energía. En 1973 la producción mundial de petróleo fue de 2.836,4 millones de toneladas y las reservas estimadas de 86.096 millones de toneladas que sólo durarian 30,35 años, hasta 2003. Para 1990 la producción mundial de petróleo 
aumentó hasta 3.257 millones de toneladas y las reservas eran de 176.478 millones de toneladas con un plazo de agotamiento de 42 años, es decir, hasta el año 2.032". Conviene anotar que, pese a la explotación de nuevos yacimientos, de 1973 a nuestros dias bemos experimentado una onda larga recesiva del Kondratief, con más años de crecimiento frenado que de crecimiento acelerado en las economias mundiales. Entre otras hay dos razones; junto con el incremento de la oferta y de las reservas de petróleo se está dando un elevado incremento de la demanda mundial, y tanto el incremento de la demanda como el de la oferta está generando mayor contaminación y degradación medioambiental, que frena directamente el crecimiento económico

Desde mediados de los años setenta entra en escena el "buracán de la globalización"; a la carrera armamentística, altamente consumidora de energías nobles e innobles, se suma la competencia económica entendida como la era del 'crecimiento bruto' (PIB), indice de la eficiencia económica. Luego de esta carrera agotadora y firmada en Minsk la desintegración de la URSS, diciembre 1991, Naciones Unidas convoca a la Cumbre de la Tierra, junio 1992. Se reúnen en Río Janeiro. Brasil, 172 gobiernos, incluidos 108 jefes de Estado y de Gobierno que aprueban tres acuerdos: la Agenda 21, la Declaración de Río sobre Medio Ambiente y Desarrollo, y una Declaración sobre los Bosques. En esta cumbre la crisis energética aparece como parte integrante de la crítica degradación medioambiental en simbiosis con el crecimiento económico y la equidad social.

La Agenda 21 tiene como fin promover un desarrollo que relacione el crecimiento económico, la equidad social y la protección del medio ambiente. Además aborda temas como la lucha contra la pobreza, protección y fomento de la salud bumana, la dinámica geográfica, protección de la atmósfera, lucha contra la deforestación, conservación de la biodiversidad y toma en cuenta "los elementos del desarrollo que afectan el medioambiente, tales como la pobreza, la deuda externa de los paises en desarrollo, los modos insostenibles de producción y consumo, la presión demográfica y la estructura de 
la economía internacional". Parecía que esta vez los gobiernos nacionales estaban dispuestos a cooperar entre si para lograr un mundo habitable.

Diez años más tarde la Declaración de Johannesburgo afirma que los paises signatarios no pusieron en marcha la Agenda 21. "Reconocemos que la erradicación de la pobreza, la protección y la gestión de los recursos naturales para el desarrollo económico y social son objetivos claves y exigencias esenciales para el desarrollo durable. La profunda brecha que divide la sociedad entre ricos y pobres y la fosa que se extiende entre los mundos desarrollados y en desarrollo constituyen la amenaza mayor para la prosperidad, la seguridad y la estabilidad mundiales. El medioambiente sigue deteriorándose". Los discursos de dos presidentes europeos bacen juego con esta declaración. Jacques. Chirac titula su discurso: "Nuestra casa está ardiendo". "Nuestra casa está ardiendo y miramos para otro lado. La naturaleza mutilada, sobrexplotada, no logra reconstruirse y nos resistimos a aceptarlo. La tierra y la bumanidad están en peligro y todos somos responsables. No podemos decir que no lo sabiamos. Tengamos cuidado de que el siglo XXI no se convierta, para las generaciones futuras, en un crimen de la bumanidad contra la vida. Queda comprometida nuestra responsabilidad colectiva. Responsabilidad, en primer lugar, de los paises desarrollados, por su historia, su poder y su nivel de consumo. Si toda la bumanidad se comportara como las naciones del Norte, harian falta dos planetas para satisfacer esas necesidades... Diez años después de Rio no tenemos mucho de qué enorgullecernos"...

Más en relación con nuestro tema, Gerbard Schröder se dirige en su discurso al gobierno de Estados Unidos: "El calentamiento de la tierra no se detiene en las fronteras de los paises. Rogamos insistentemente a los Estados miembros de Naciones Unidas que ratifiquen lo antes posible el protocolo de Kyoto y cumplan con sus objetivos. Me dirijo, sobre todo, a Estados Unidos de América para que asuma su responsabilidad en la protección del clima y colabore igualmente en la reducción de gases de efecto invernadero. Esperamos que Estados Unidos se integre de nuevo a los acuerdos 
internacionales sobre protección del clima. Una política energética durable permite conciliar la conservación de los recursos naturales con el desarrollo económico sostenido. Sabemos que los paises en desarrollo no pueden, por sí solos, echar a andar una estrategia energética tan ambiciosa. Por ello y junto con el resto de Estados europeos defendemos la ampliación de un mecanismo central para la protección mundial del medio ambiente. Presento tres propuestas concretas para propiciar, a escala mundial, el uso de energias renovables, a ejemplo de la Conferencia Internacional de Bonn sobre el agua dulce: elaborar una estrategia internacional para el desarrollo de estas energías. Propongo que una institución de Naciones Unidas tenga como encargo concreto ayudar, sobre todo a países en desarrollo, a crear un sistema de aprovisionamiento de energia limpia, dando prioridad a las energias renovables. Alemania firmará con los países en desarrollo y emergentes acuerdos estratégicos con objetivos concretos adaptados a su respectiva situación, que se realicen conjuntamente". ("La cumbre de la tierra en su entorno mundial". ECA, 2002, pp. 771-784)

Alemania y Francia propusieron que los delegados a la cumbre firmaran el compromiso de que "el 15\% de la energía total fueran energías limpias", eólicas, solares..., pero encontraron la oposición de países productores de petróleo, entre ellos Estados Unidos. Fuera de compromiso concreto la Unión Europea presentó oficialmente una iniciativa política para promoverlas. Una amplia lista de países ba suscrito la iniciativa: Bulgaria, Chipre, Chequia, Paises Bálticos, Hungría, Islandia, Malta, Nueva Zelanda, Noruega, Polonia, Rumania, Eslovaquia, Eslovenia, Suiza, Turquia, varios paises de América Latina y, al parecer, India y Brasil. Esta lista es interesante para nuestro tema, ya que son muchos los paises que no quieren que las energías limpias queden en la vía muerta. (Ibidem; p. 180) Pese a todas las rogativas, el actual gobierno de Estados Unidos ni se ha dignado leer la obra de Al Gore, La tierra en juego, ni entonces ni abora ha aceptado ratificar el protocolo de Kyoto. 


\section{Del petróleo a la energía nuclear}

Todos sabemos que la dinastía Bush está muy ligada al grupo petrolero norteamericano, a comenzar por la quebrada empresa Enron, cuyo director Ken Lay ba sido amigo cercano de G. W. Bush; el vicepresidente Dick Cheney fue director de la empresa Halliburton especializada en apagar incendios de pozos petroleros, $y$ Condoleeza Rice es el nombre de un barco petrolero. Paul $O^{\prime}$ Neil, primer Secretario del Tesoro del presidente Bush, afirmó baber tenido en sus manos, en enero de 2001, un documento calificado de secreto: "Un plan para Irak después de Saddam Hussein; enviar tropas de paz, organizar tribunales de justicia y recuperar las riquezas petroleras de Irak". Ocho meses antes del 11 de septiembre ya estaba planeada la invasión de Irak, porque, con fecha 6 de noviembre de 2000, el gobierno de Saddam Hussein habia trasladado sus activos multimillonarios de la zona dólar a la zona euro. Mientras el petróleo sea la alternativa principal para la generación de energía es lógico que sus elevados precios sean beneficiosos para sus productores y vendedores, al mismo tiempo que se traducen en estanflación (crecimiento frenado con alza de precios) para los demandantes y no productores del "oro negro".

Abora el presidente G. W. Bush se ba pasado a este segundo grupo al percibir la intensidad de la crisis energética en su propia nación. "Millones de familias norteamericanas y de pequeñas empresas sufren a causa del alza de precios de la gasolina. Mi gobierno va a hacer todo lo posible para tener unos precios más asequibles. Voy a animar a los paises productores de petróleo a aumentar la producción", para que desciendan los precios. Bush prometió "proteger a los consumidores, de manera que no baya manipulación de precios en las estaciones de servicio del combustible... Esto es como un impuesto sobre las pequeñas empresas que crean empleos. Es un impuesto sobre las familias que afecta su moral. Creo que todo esto está afectando la marcha de la economía".

Recordando que la demanda de energía en Estados Unidos ba aumentado "cuarenta veces más rápido que la producción de energia", Bush propone un plan para reducir la dependencia del 
extranjero, reconociendo que hará falta un tiempo de espera. "Hay que modernizar la industria del petróleo. Un futuro energético seguro para Norteamérica depende de la construcción y desarrollo de refinerías de petróleo norteamericanas". Bush recordó que no se ba construido ninguna refinería en Estados Unidos desde 1976. "Esto significa que gastamos cada vez más en importaciones de productos refinados. Para animar el desarrollo de posibles yacimientos el ministerio de Medio Ambiente debe simplificar algunas reglamentaciones". ("Bush promete hacer bajar los precios de la gasolina”. Le Monde, 29 de abril de 2005)

Abora el gran defensor del libre mercado juega el papel de "defensor del consumidor" y para ello propone una nueva fuente de energía limpia y segura: "El futuro energético de Estados Unidos incluye ampliar la energía nuclear. La energía nuclear proporciona sólo un 20\% de la electricidad en Estados Unidos, sin polución atmosférica y sin emisión de gases de efecto invernadero. Es una de las energias más seguras y limpias del mundo y tenemos que usarla más. Por desgracia, Norteamérica no ba construido ninguna central nuclear desde 1970. En el mismo tiempo Francia ba fabricado 58 reactores y el $78 \%$ de su electricidad limpia y segura viene de la energía nuclear. Estados Unidos debe construir nuevas centrales nucleares".

Bush es una persona que, desde su rincón tejano, ha estado más relacionado con el medio petrolero que con el mundo científico de la energía nuclear. Esto da a pensar que detrás del presidente Bush un grupo de cientificos propone y apoya esta alternativa. Las razones pueden ser múltiples, entre ellas impulsar una linea de inversión directa en el extranjero. Por ello Bush ba insistido en el desarrollo de nuevas fuentes de energía, sugiriendo animar a paises emergentes como China e India - grandes consumidores de energia - a utilizar las tecnologias más nuevas y más eficaces, en orden a reducir la demanda mundial de energias fósiles. Igualmente Bush ba becho presión sobre Arabia Saudita para que aumente la producción del crudo y asi puedan bajar los precios. ("George W. Bush promete impulsar la energía nuclear”. Le Monde, 28 de abril de 2005) 
La propuesta de la energía nuclear como una alternativa complementaria al petróleo plantea una serie de interrogantes. Si un país como Estados Unidos, que tiene petróleo en su subsuelo, está sufriendo en millones de familias y de empresas las consecuencias económicas y sociales de la prolongada crisis energética, esto es una prueba manifiesta de "la pesada losa de deuda externa" que grava a las pequeñas economias no productoras de petróleo. Debemos pensar que esta presión de los años 2004-2005 puede prolongarse por más tiempo sin que una bola de cristal nos pueda decir basta cuando. Dado que este problema se está haciendo mundial es de esperar que estas propuestas de Bush no queden flotando en el vacio. Por ejemplo, a final del mes de mayo, Paul Wolfowitz, amigo de Bush, sustituye a James Wolfensobn en la presidencia del Banco Mundial. Por supuesto que la mayoría de naciones de África y América Latina no están técnicamente capacitadas para derivar su electricidad de la energía nuclear; sin embargo, Paul Wolfowitz podría pasar a la historia como un reconocido humanista si invita a China e India, poseedores de la energía atómica, a obtener un elevado porcentaje de su energía civil a partir de la energía nuclear, permitiendo que el resto de paises del tercer mundo dispongan de las energías fósiles a precios más cómodos.

Donde se ba discutido este problema, en forma apasionada, ba sido en la reunión de Naciones Unidas, celebrada los dias 2 y 3 de mayo 2005, dedicada a la revisión del Tratado de No Proliferación Nuclear (TNP). Valga recordar que la 'energía nuclear' se desarrolla en muchos paises con fines civiles de generación de electricidad, mientras que la 'energía atómica' se ba desarrollado en muchos países como arma disuasiva (excepto el caso de Hiroshima y Nagasaki) entre potencias que siguen practicando la guerra fría. Ambas energias tienen en común que son altamente contaminantes del medio ambiente, más la segunda que la primera.

\section{Los residuos nucleares}

No deja de ser atractiva la propuesta hecha por el presidente Bush en su discurso a la nación, porque si Estados Unidos y otros países elevan por encima del $20 \%$ el monto de electricidad generada 
con energía nuclear, ciertamente aumentaría la disponibilidad de recursos fósiles para países más necesitados y endeudados. También uno se pregunta por qué serie de razones Estados Unidos, con sus laboratorios y científicos, y con su programa del escudo antibalístico, no ba desarrollado más allá del $20 \%$ de su electricidad a partir de energía nuclear y no ba construido un reactor nuclear desde 1970. ¿Por qué cita el ejemplo de Francia y no la ban imitado ni Bush padre ni Bush bijo? Hacemos la pregunta sin saber cuál pueda ser la respuesta, aunque intuimos su razón de ser.

Por curiosidad hemos ojeado algunos apuntes de Greenpeace sobre residuos nucleares y bastan unos breves párrafos para entender los riesgos de contaminación de esta alternativa "limpia y segura", que decía Bush. "Se producen residuos nucleares en cada una de las etapas del ciclo del combustible nuclear, desde la minería del uranio al reprocesamiento del combustible nuclear irradiado. La mayor parte de la basura nuclear permanece siendo peligrosa por miles de años, dejando asi una peligrosa berencia a las futuras generaciones... Como parte de su operación rutinaria, toda central nuclear descarga algunos de sus materiales directamente al ambiente. Algunos residuos líquidos se descargan con el agua de refrigeración de las turbinas hacia el mar, ríos o lagos, y otros residuos gaseosos se liberan a la atmósfera... De acuerdo a estimaciones hechas por la Agencia Internacional de Energía Atómica (AIEA), la cantidad global de combustible nuclear en la actualidad ronda las 200.000 toneladas. Se cree que llegará a unas 450.000 toneladas para mediados del siglo XXI. A pesar de que se ban estudiado una variedad de métodos para disponer de estos residuos durante las últimas décadas -incluido arrojarlos al espacio- no existe aún una solución acerca de qué hacer con esta basura nuclear. La mejor solución para el futuro es que no debería producirse más basura nuclear a nivel mundial. Durante las próximas tres décadas más de 350 reactores nucleares deberán salir de servicio. Ya van más de 40 años desde que el primer reactor comercial comenzó a producir electricidad y la industria no tiene respuesta sobre cómo bacer seguro $y$ económico el desmantelamiento de un reactor". 
No es necesario agregar más datos sobre el peligro de contaminación mortal de los residuos nucleares, aunque se nos podrá decir que hemos sido demasiado alarmistas. Por otra parte, lo que pudiera ser una 'ruta de concertación', es decir, definir qué porcentaje de energía civil (electricidad) es aconsejable y permisible generar a partir de la energía nuclear, se ba convertido en punto de confrontación, que se deberá resolver en Naciones Unidas. Como indica el diario El País, "El tratado de no proliferación queda al borde del fracaso ante el desafío nuclear". Ahora Estados Unidos vuelve a estar en mala posición cuando, luego de atacar a Irak, amenaza a Irán porque desarrolla el proceso del uranio enriquecido para disponer de energía nuclear con fines civiles. Si G. W. Bush propone desarrollar la energía nuclear en Estados Unidos para uso civil y la está recomendando a China y a la India como una gran alternativa a la crisis energética mundial, ¿̇con qué derecho el presidente de Estados Unidos, por mediación de Condoleeza Rice, puede presionar para que la trilogia de Alemania, Francia $e$ Inglaterra se oponga a que Irán desarrolle la energía nuclear con fines civiles, cuando los paises que la circundan, Israel, India, Pakistán, China y Corea del Norte, han desarrollado el arma nuclear? Estas presiones pueden resultar contraproducentes porque Estados Unidos no bubiera invadido Irak si Saddam Hussein dispusiera del arma atómica. Estados Unidos está convirtiendo una posible solución científica en una confrontación política.

Es un problema delicado, porque el pasado 3 de mayo, en plena sesión de la ONU, Irán anuncia públicamente "su determinación de proseguir sus actividades en todo el ámbito legal de la tecnología nuclear, incluido el enriquecimiento de uranio, exclusivamente con fines pacíficos". ("Irán decide proseguir el enriquecimiento del uranio". Le Monde, 3 de mayo de 2005) Los técnicos dicen que el uranio débilmente enriquecido proporciona el combustible necesario para las centrales nucleares de uso civil; pero el uranio altamente enriquecido puede servir para la fabricación de armas nucleares". (El País, 3 de mayo de 2005) ¿Quién suelta este nudo gordiano? Durante los cuatro primeros meses de 2005, G. W. Bush y sobre 
todo Condoleeza Rice, temen que si Irán desarrolla el sistema de uranio enriquecido no se quede en el primer peldaño, si no que pase a la gestación de energía atómica y que Irán pueda ceder a alguna de las facciones de Al Qaeda que amenazan a Estados Unidos. El gobierno de Irán ba repetido que "Irán no es Corea del Norte" y que ellos admiten las inspecciones del Organismo Internacional de Energía Atómica. Su director, Mabomed el Baradel, "advirtió a Estados Unidos que un ataque militar en territorio irani sólo empeoraría las cosas".

No sabemos todavía qué determinación tomará el Consejo de Seguridad de Naciones Unidas ante esta decisión del gobierno de Irán. Muchas personas juzgan que el nombramiento de Jobn Bolton, como delegado con poder de veto en el Consejo de Seguridad, no ha sido la más acertada. Parecería que la administración Bush quiere - a su guisa- abrir y cerrar la puerta a la generación de energía civil a partir de energía nuclear, dando ellos el banderillazo de salida. Si tomamos en cuenta que $G$. W. Bush ba reformateando su concepto de terrorismo por el de tirania, y Condoleeza Rice amplía la lista de Irán y Corea del Norte introduciendo a Cuba, Birmania, Bielorrusia, Zimbabwe, Chechenia, Tibet, como posibles canales de transmisión del arma nuclear a alguna de las facciones de Al Qaeda, tendríamos que la fuente de energía nuclear ya no es limpia ni segura. Con su discurso del 28 abril, G. W. Bush nos abria la puerta a una curiosidad: iquerrá realmente buscar una alternativa a la crisis energética a partir de la cuestionada fuente de la energía nuclear?

Mirando bacia atrás, se llega a la conclusión de que no es esa la via que lleve a la alternativa mejor. Siendo el mayor contaminante mundial el actual gobierno de Estados Unidos ba mantenido una política negativa e islacionista ante el reto de la degradación ambiental, aduciendo que el protocolo de Kyoto es malo para su economía. La solución hay que buscarla desde un debate abierto en Naciones Unidas, que ya está formulado desde las cumbres de la Tierras 1992 y 2002. El próximo mes de septiembre deben discutirse las grandes reformas de Naciones Unidas presentadas por el Grupo 
de Sabios, de manera especial la estructura del Consejo de Seguridad. Desde esta perspectiva deseamos actualizar la propuesta presentada por G. Schröder en Jobannesburgo: "Una conferencia internacional sobre energias renovables, a ejemplo de la Conferencia Internacional de Bonn, sobre el agua dulce: Elaborar una estrategia internacional para el desarrollo de estas energías. Propongo que una institución de Naciones Unidas tenga como encargo concreto ayudar sobre todo a países en desarrollo a crear un sistema de aprovisionamiento de energía limpia, dando prioridad a las energias renovables"... Nos parece que ésta es una alternativa más limpia y segura, aunque tengamos que esperar hasta el mes de septiembre. 